\title{
Variability in General Activity and the Expression of Complex Behavior in the Fetal Rat (Rattus norvegicus)
}

\author{
James L. Reilly and Steven S. Robertson \\ Cornell University
}

\author{
Benjamin D. MacLennan \\ and William P. Smotherman \\ Binghamton University-SUNY
}

\begin{abstract}
Rat fetuses exhibit intrinsic fluctuations in general motor activity and respond to an artificial nipple (AN) with mouthing and oral grasping behavior. The present study examined the relation between the organization of general activity and the expression of these specific responses to an AN on Embryonic Day 21. In Experiment 1, continuous exposure to the AN resulted in nonspecific behavioral activation characterized by an increase in amplitude and high-frequency variability. In Experiment 2, increased amplitude and variability in general activity preceding discrete presentations of the $\mathrm{AN}$ resulted in more mouthing and oral grasping responses to the AN. These results suggest that presentation of the AN triggers behavioral reorganization in which the level and variability of overall activity may facilitate expression of well-defined action patterns.
\end{abstract}

A principal aim of the neuroembryological study of behavior has been to examine behavioral patterns that emerge in the embryo and fetus amid continuous nervous system development, organogenesis, and environmental influence (Oppenheim, 1982). Investigations of embryonic and fetal behavior have sought to illuminate the role of activity in neurobehavioral development. Typically, prenatal behavior has been conceptualized as essential to normative development and postnatal behavior, as an ontogenetic adaptation to conditions within the prenatal environment or as an epiphenomenon reflecting central nervous system functioning at a given time in development (Oppenheim, 1981, 1984). As research on behavior during early development has advanced, it has become obvious that fetal behavior both serves adaptive functions in the prenatal environment and prepares the organism for postnatal life (Oppenheim, 1981, 1984; Smotherman \& Robinson, 1990).

Since the work of Preyer (1937), embryonic motility has been studied in numerous vertebrates (e.g., Bekoff, 1981a, 1981b; Hamburger, 1963, 1973; Hooker, 1952), and its ubiquity has raised intriguing questions for comparative

James L. Reilly and Steven S. Robertson, Department of Human Development, Cornell University; Benjamin D. MacLennan and William P. Smotherman, Department of Psychology, Binghamton University-SUNY.

This research was supported by National Institute of Child Health and Human Development Grants HD23814 and HD28014 and by National Institute of Child Health and Human Development Merit Award HD16102 and Grant HD28014. We thank Wayne Kashinsky for help in instrument design and Lora Becker, Kristyn Snyder, and Elena Varlinskaya for help in data collection.

Correspondence concerning this article should be addressed to Steven S. Robertson, Department of Human Development, MVR Hall, Cornell University, Ithaca, New York 14853, or to William P. Smotherman, Department of Psychology, Binghamton UniversitySUNY, Box 6000, Binghamton, New York 13902-6000. Electronic mail may be sent via Internet to ssr $4 @$ cornell.edu or to smother@binghamton.edu. behavioral science. Research on both spontaneous activity and behavioral responsiveness to multiple forms of stimulation in the fetus has yielded a greater appreciation of the complexity of fetal behavior and the role that the fetus plays in its own development (Smotherman \& Robinson, 1988). Whereas advances in real-time fetal monitoring devices (e.g., ultrasound imaging) have permitted both quantitative and qualitative views of activity in the human fetus (de Vries, Visser, \& Precht1, 1982, 1985), methodological advances in the investigation of other mammalian fetuses (e.g., rodents and sheep) have allowed direct observations of fetal behavior and the opportunity for experimental manipulations. One animal model that has proven to be highly accessible for developmental psychobiological investigations is the fetal rat (Smotherman \& Robinson, 1987b, 1991, 1996).

Narayanan, Fox, and Hamburger (1971) provided the first comprehensive investigation of motility in the fetal rat. They reported that spontaneous movement appeared randomly intermittent and aimless in that it lacked resemblance to the integrated action patterns characteristic of postnatal behaviors (Narayanan et al., 1971). Recent investigations of fetal activity in the rat have involved more sensitive measures, and these studies have used both quantitative and qualitative analyses and have established evidence for temporal and spatial organization of spontaneous activity (Bekoff \& Lau, 1980; Robinson \& Smotherman, 1987; Smotherman, Robinson, \& Robertson, 1988). The development of speciestypical behavioral patterns in response to sensory stimulation also has been documented (Robinson et al., 1992; Smotherman \& Robinson, 1987a). It has been suggested that the randomness of spontaneous activity early in gestation is replaced by progressive levels of organization, thus forming the foundation from which postnatal behavior is built (Robinson \& Smotherman, 1988).

The development of motor synchrony in the fetal rat is one of the earliest forms of organization, with an increase in synchronous movements among different body regions 
during the last days of gestation (Embryonic Days 18-21 [E18-E21]; Robinson \& Smotherman, 1992b). The spontaneous activity of the fetus during late gestation also exhibits a considerable degree of temporal organization. Cyclic motility similar to that described in the fetal human (Robertson, 1985) and sheep (Robertson \& Bacher, 1995) has been documented in the fetal rat on E20 (Smotherman et al., 1988). Highly organized motor activity in the fetal rat is expressed in response to sensory stimulation. During the last days of gestation, presentation of both chemosensory and tactile stimuli reliably elicits coordinated responses in the fetal rat, including components of contact righting, stepping, punting, suckling, and grooming behaviors (Robinson \& Smotherman, 1992a).

Organization in fetal motor activity has been documented, but little is understood about how variation in motor activity may affect responses to stimulation during behavioral development. The aim of the present study was to examine how the temporal organization of spontaneous activity is related to the expression of organized responses to an artificial nipple (AN) that approximates the features of the lactating female rat's nipples. Tactile stimulation around the perioral region by an AN reliably elicits mouthing and licking of the AN that leads to expression of the oral grasp response. This collection of motor patterns resembles components of postnatal suckling behavior (Robinson et al., 1992). The present study recorded the motor activity of the E21 rat fetus. Comparisons between unmanipulated fetuses and fetuses continuously exposed to the AN provided information about how AN stimulation changed the overall organization and variability of motor activity across time. Analyses within fetuses exposed to multiple, discrete presentations of the AN determined whether different properties of motor activity could predict the response elicited by the AN.

\section{General Method}

\section{Subjects}

Fetal subjects were the offspring of time-mated Sprague-Dawley rats (Charles River Laboratories, Wilmington, MA). Breeding females were housed in groups of 3 in plastic breeding cages $(36 \times 47 \times 20 \mathrm{~cm})$ and maintained under conditions of constant room temperature $\left(22^{\circ} \mathrm{C}\right)$ on a 12-hr light-dark cycle (lights on at 0700 ). Food and water were available ad libitum. Daily vaginal smears were taken during a 4-day breeding period to identify the date of conception; E0 was defined as the 1st day of detectable sperm (birth occurs on E21.5). Procedures for the care and use of adult and fetal rats were in accordance with the guidelines established by the National Institutes of Health (1986).

\section{Preparation of Pregnant Females and the Fetal Environment}

On E21, pregnant females were placed under light ether anesthesia so that a chemomyelotomy could be performed. Females received an injection of $70 \mu 100 \%$ ethanol into the spinal cord between the first and second lumbar vertebrae. This procedure produces an irreversible spinal blockade in the lower thoracic and lumbosacral regions (Smotherman, Richards, \& Robinson, 1984). The prepared female was placed in a Plexiglas holding apparatus and partly submerged in a temperature-regulated $\left(37 \pm 0.5^{\circ} \mathrm{C}\right)$ water bath containing an isotonic saline solution. A low midline laparotomy was performed, and the uterus was exteriorized into the surrounding bath. A 20-min acclimation period was allowed after which individual fetuses were delivered through a small incision in the uterine wall and the amniotic and chorionic membranes were removed. No more than 2 fetuses from a female were tested. When 2 subjects were used, they were assigned to different treatment conditions.

Fetal subjects remained submerged in the saline bath with the placental and umbilical attachments intact and were supported by a plastic spoon during testing. The condition of the fetus was monitored continuously throughout the experiment, and only those fetuses that maintained a healthy coloration (indicating sufficient oxygen supply) were used as subjects. All experimental trials took place between $0900 \mathrm{hr}$ and $1200 \mathrm{hr}$. At the conclusion of an experiment, adult females and fetuses were humanely killed by rapid cervical dislocation. These methods have been used extensively in previous studies of fetal rat behavior (Smotherman \& Robinson, 1991).

\section{Recording of Gross Motor Activity}

A recording electrode was constructed from the shank of a 25 gauge needle attached to a copper wire (diameter: $0.15 \mathrm{~mm}$ ). The electrode was implanted under the skin on the midline of the subject's back when the subject was externalized from the uterus and surrounding membranes $(20 \mathrm{~min}$ before the start of the test session). The electrode was connected to a custom-made, high-gain instrument amplifier. Movement detected by the electrode was sampled at $15 \mathrm{~Hz}$ and digitized on-line throughout the experimental session. This recording system provides a measure of overall fetal motor activity. This measure of overall motor activity was collected continuously during the test session. Data were collected for a 30-min period unless fetal or maternal health became compromised (e.g., poor fetal coloration, placental rupture, or uterine bleeding). Only data from healthy fetuses were included in analyses.

\section{Presentation of the AN and Recording of Mouthing and Oral Grasping Responses}

The AN was fashioned from soft vinyl material with dimensions similar to those of a lactating female rat's nipple. The base of the AN was attached to a handle allowing manual presentation by the experimenter. Presentation of the AN consisted of continuous, gentle contact with the perioral region. Movement of the AN was limited to small adjustments to maintain contact between the AN and the perioral region of the subject fetus. Movement of the AN did not cause the fetus to be moved or create fluid currents. No attempt was made to force the tip of the AN into the mouth of the subject (Robinson et al., 1992).

A video camera (Canon Super Macro AF 6-60 mm) positioned $65 \mathrm{~cm}$ directly above the fetal subject provided a video record (at 30 frames per second) of the experimental session. The video record was synchronized with the recording of overall fetal activity. The video recordings were coded for mouthing and the oral grasping responses. Mouth movements consisted of the opening and closing of the mouth in response to the AN presentation. A mouth movement was coded when it occurred in at least two consecutive video frames. Mouth movements that occurred within $2 \mathrm{~s}$ of one another (i.e., the end of one continuous mouthing movement was followed by the onset of another continuous mouthing movement in less than $2 \mathrm{~s}$ ) were coded as a single mouth movement. The oral grasp response consisted of the mouth 
opening, the AN entering the oral cavity, and the mouth closing around the tip of the AN (Robinson et al., 1992).

\section{Experiment 1: Continuous Presentation of AN}

In Experiment 1, E21 rat fetuses received a continuous presentation of the AN. Overall motor activity was analyzed to determine the effects of continuous perioral stimulation from an AN on the amount, variability, and temporal organization of spontaneous motor activity.

\section{Method}

Subjects and procedure. Twenty-nine fetuses from 22 pregnancies were assigned to one of two conditions. Fetuses in the unmanipulated condition $(n=14)$ were not exposed to the AN. These control fetuses provided baseline information on the amount, variability, and temporal organization of spontaneous motor activity. Fetuses in the continuous presentation condition $(n=15)$ were exposed continuously to the AN.

Data analysis. Before any analyses of the activity time series, a moving minimum calculated over a 5-s window was subtracted from the unprocessed data to remove baseline instability. The resulting time series was integrated in nonoverlapping 2 -s windows (Robertson et al., 1996). The average level of activity was calculated for each subject. The average of the absolute values of the point-to-point differences ( 2 -s windows) in the activity time series was calculated to provide a measure of variability.

As a means of measuring the temporal characteristics of overall activity more precisely, the data from each fetus were integrated over a 1-s window and then spectral analyzed to identify and quantify cyclic organization. The activity time series was detrended, and the Fourier transform of the autocorrelation function was calculated with an algorithm written by Jenkins and Watts (1968). A Tukey lag window with a bandwidth of 0.32 cycles per minute was used. Computed this way, the movement spectrum provides statistically stable estimates of the relative strength of periodic fluctuations occurring at different frequencies. The proportion of movement variance within a given frequency band is computed by the area under the movement spectrum between the two frequencies. Cyclic organization was inferred from a peak in the movement spectrum exceeding the upper $99 \%$ confidence limit of white noise. This analysis has been used in previous investigations of the cyclic properties of spontaneous activity (Robertson, 1985; Smotherman et al., 1988). Four measures of cyclic organization were computed for each activity spectrum that contained a significant peak: (a) The frequency of the largest peak indicated the dominant rate of oscillation in overall activity; (b) the height of the largest peak indicated the strength of the dominant oscillation in activity; (c) the width of the largest peak at its half-maximum point indicated the irregularity of the dominant oscillation; and (d) the proportion of variance due to fluctuations greater than 3 cycles per minute was derived by computing the area under the activity spectrum above 3 cycles per minute.

\section{Results}

The lengths of the experimental sessions (mean \pm standard error of mean) were similar in the unmanipulated $(28.1 \pm 1.3$ min) and continuous AN exposure $(26.7 \pm 1.7 \mathrm{~min})$ conditions. There were small decreases (approximately 10\%) in overall activity in both groups between the first and second halves of the experimental session: unmanipulated condi- tion, $33.8 \pm 4.0$ versus $30.1 \pm 2.7, t(13)=1.42, p=.18$ and continuous AN exposure condition, $63.7 \pm 6.1$ versus $57.8 \pm 5.9, t(14)=3.11, p=.008$.

Overall activity was higher in the continuous exposure condition than in the unmanipulated condition, $t(27)=4.14$, $p<.001$ (see Figure 1, top). The average absolute point-topoint difference in activity also was greater in the fetuses continuously exposed to the AN than in the unmanipulated fetuses, $t(27)=3.38, p=.002$ (see Figure 1, bottom).

Spectral analysis revealed cyclic fluctuations in the activity of all fetuses in both conditions, indicated by the presence of a significant peak in the spectrum. Activity time series and spectra from 2 fetuses (1 in the unmanipulated condition and 1 in the continuous exposure condition) are shown in Figure 2. Fetuses in the continuous exposure condition exhibited faster dominant oscillations in activity ( $3.66 \pm 1.25$ cycles per minute) than unmanipulated fetuses $(1.00 \pm 0.23$ cycles per minute), $t(27)=2.03, p=.05$ (see Figure 3, top left), although the dominant oscillations were slightly weaker (continuous exposure, $0.13 \pm 0.02$ cycles per minute ${ }^{-1}$; unmanipulated, $\left.0.19 \pm 0.02\right), t(27)=1.97$, $p=.06$ (see Figure 3, top right). Continuous exposure to the AN did not, however, influence the irregularity of the dominant fluctuations (continuous exposure, $0.83 \pm 0.05$ cycles per minute; control, $0.93 \pm 0.07), t(27)=1.23, p=$ .23 (see Figure 3, bottom left). The proportion of activity variance due to fluctuations faster than 3 cycles per minute
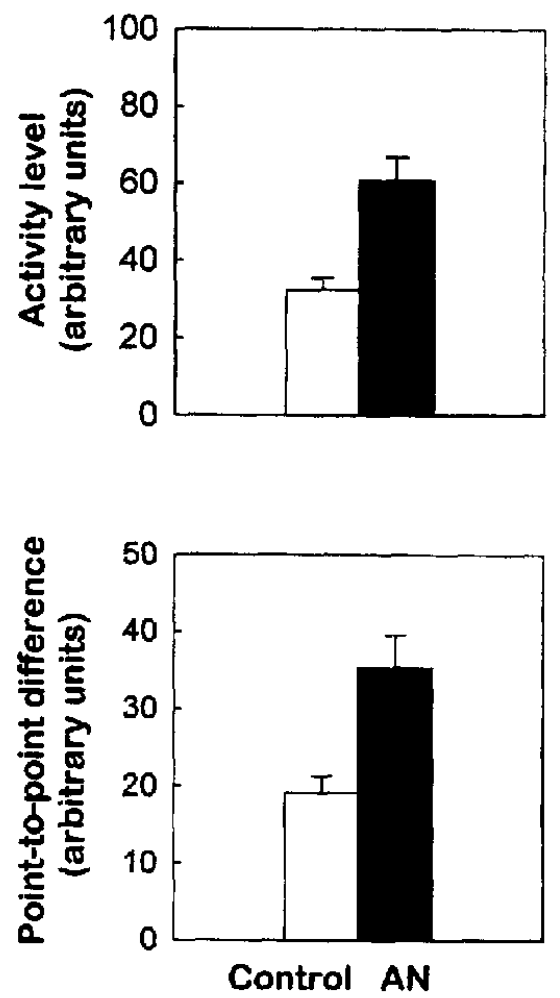

Figure 1. Mean ( $\pm S E M$ ) level (top) and absolute point-to-point difference (bottom) of the 2-s integrated activity time series of control fetuses and fetuses continuously exposed to an artificial nipple (AN). 

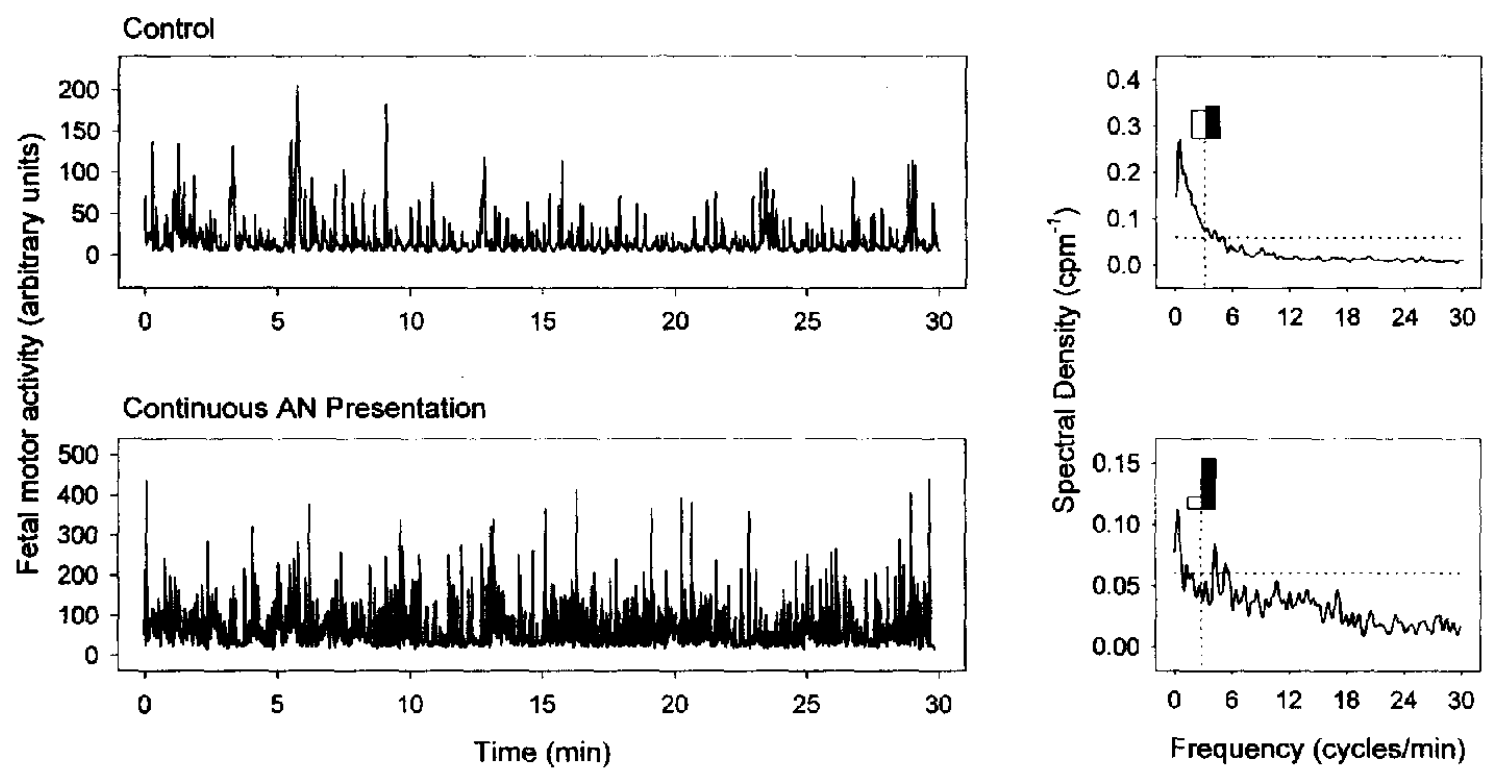

Figure 2. Individual 1-s integrated activity time series (left) and spectra (right) for 2 fetuses. The horizontal line in the spectra is the upper $99 \%$ confidence limit for the spectral estimates of a white noise. The bars indicate the relative proportions of variance accounted for by fluctuations slower (open bars) and faster (solid bars) than 3 cycles per minute (cpm). $\mathrm{AN}=$ artificial nipple.

was greater in continuous exposure fetuses $(.77 \pm .02)$ than in unmanipulated fetuses $(.64 \pm .02), t(27)=4.64, p<.001$ (see Figure 2 and Figure 3, bottom right). Within the continuous exposure group, the level of activity, its point-topoint variability, the frequency of the dominant oscillation, and the strength of the dominant oscillation were not related
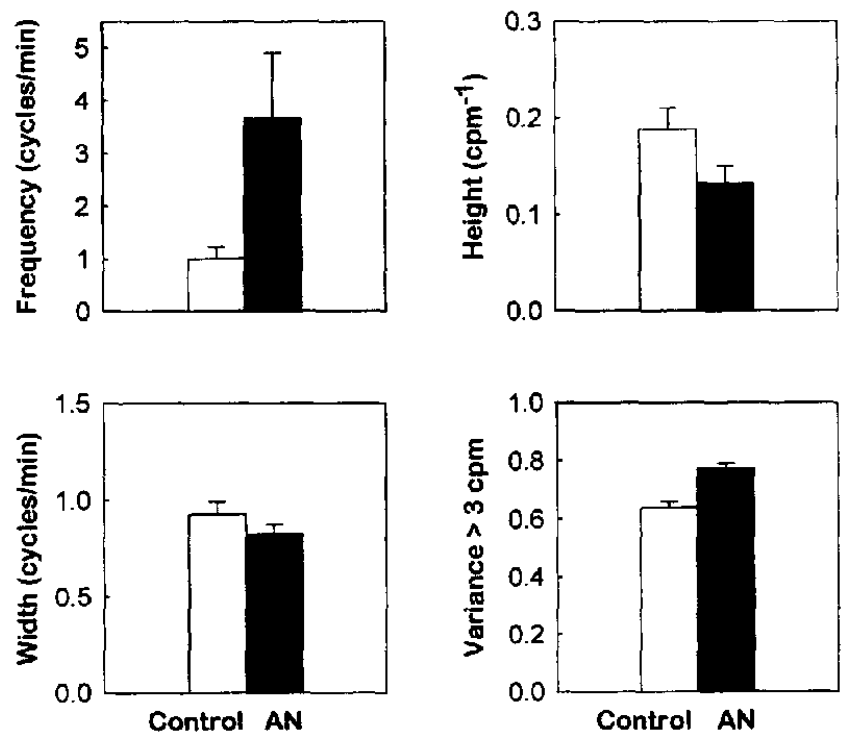

Figure 3. Means ( $\pm S E M$ ) for the frequency, height, and width of the primary peak in the activity spectrum, along with the proportion of variance due to fluctuations faster than 3 cycles per minute (cpm), for control fetuses and fetuses continually exposed to an artificial nipple (AN). (linear or quadratic relation) to the rate of mouthing or oral grasping (all $p$ values $\geq .09$ ).

\section{Discussion}

The average absolute point-to-point difference, as well as the results of the spectral analysis, revealed that continuous presentation of the AN increased the high-frequency fluctuations of activity. In addition, AN exposure increased the level of activity, which is consistent with reports of increased activity resulting from shorter periods (1 min) of AN exposure (Becker \& Smotherman, 1996). None of the changes in motor activity induced by continuous presentation of the AN were attributable to mouthing and grasping. Taken together, these results suggest that continuous presentation of the AN triggers nonspecific behavioral activation characterized by an increase in the level and high-frequency variability of overall motor activity.

\section{Experiment 2: Discrete Presentations of the AN}

In Experiment 2, E21 rat fetuses received discrete presentations of the AN. The 30-s periods preceding presentations that resulted in no response, mouthing without grasping, or oral grasping were compared. On the basis of the results of Experiment 1, the analyses in the second experiment focused on the level of and variability in overall activity.

\section{Method}

Subjects and procedures. Fourteen fetuses from eight pregnancies were tested. A 4-min period at the beginning of the session during which there was no $\mathrm{AN}$ exposure was used to estimate 
baseline activity level for each subject. On-line analysis of overall activity signaled the experimenter to present the AN when activity (2-s moving average) was in the lower third of the baseline range for 5 consecutive seconds and $45 \mathrm{~s}$ had elapsed since the end of the preceding AN presentation. Presentation of the AN when activity was low permitted immediate contact between the AN and the perioral region of the test subject and controlled the level of activity at the moment of AN presentation. Each discrete presentation of the AN lasted for $15 \mathrm{~s}$. Figure 4 depicts the experimental time line.

Data analysis. As in Experiment 1, a 5-s moving minimum was subtracted from the unprocessed data to remove baseline instability, and the time series was then integrated in nonoverlapping 2-s windows. For the $30-$ s period preceding each discrete AN presentation, the average level of activity and the average absolute point-to-point difference in activity were calculated. Each of the discrete presentations of the AN was classified into a category according to the subject's response to the AN presentation: (a) no response, (b) mouthing without grasping, or (c) oral grasping.

The results from multiple presentations for a given subject were averaged within a category before the data were analyzed. Three fetuses had fewer than two instances of one or more of the categories and therefore were not used in the analyses, resulting in a sample size of 11 . Separate within-subject analyses of variance were used to compare level of overall activity and absolute point-to-point difference in activity during the 30 -s period preceding AN presentation according to the subject's response to the AN. The length of the prestimulus period ( $30 \mathrm{~s}$ ) was too short to justify spectral analysis.

\section{Results}

The average length of the session in Experiment 2 was $27.9 \pm 1.1 \mathrm{~min}$. The proportion of $\mathrm{AN}$ presentations that elicited grasping did not differ between the first and second halves of the experimental session (.27 $\pm .04 \mathrm{vs.} .20 \pm .05)$, $t(10)=1.28, p=.23$, nor did the proportion of $\mathrm{AN}$ presentations that elicited mouthing (.51 $\pm .05 \mathrm{vs} . .41 \pm .05)$, $t(10)=1.67, p=.13$.

Analysis of variance revealed that the level of activity differed according to the behavioral response that followed, $F(2,20)=6.94, p=.005$. Activity level was greater before presentations that evoked mouthing $(68.1 \pm 6.0)$ and grasping $(75.0 \pm 6.5)$ than before presentations that evoked no response $(59.7 \pm 5.4)$ : mouthing versus no response, $t(10)=2.19, p=.05$, and grasping versus no response, $t(10)=3.44, p=.006$. The level of activity before presentations that elicited mouthing did not differ from the level of activity before presentations that elicited grasping, $t(10)=1.71, p=.12$.

The absolute point-to-point difference in activity also differed according to the behavioral response that followed, $F(2,20)=8.66, p=.002$. The absolute point-to-point differences preceding AN presentations that elicited mouthing $(42.8 \pm 4.5)$ or grasping $(48.8 \pm 5.2)$ were greater than the absolute point-to-point difference preceding AN presentations that did not evoke mouthing or grasping (36.8 \pm 4.1 ), $t(10)=2.21, p=.05$, and $t(10)=3.59, p=.005$, respectively. Furthermore, the absolute point-to-point difference in activity preceding AN presentations that evoked grasping was greater than the absolute point-to-point difference in activity preceding $\mathrm{AN}$ presentations that evoked only mouthing, $t(10)=2.37, p=.039$.

Because the 30 -s periods could also be grouped according to the behavioral category that they followed (rather than that which they preceded), the analyses were repeated with the $30-\mathrm{s}$ periods grouped by the behavioral response that occurred during the preceding AN presentation. Grouped this way, neither the level nor the absolute point-to-point difference in activity differed according to behavioral category, $F(2,20)=0.50, p=.61$, and $F(2,20)=0.47$, $p=.63$, respectively.

\section{Discussion}

The results of Experiment 2 demonstrate that the type of behavioral response elicited by the AN differed according to the level and absolute point-to-point difference in activity during the $30 \mathrm{~s}$ preceding discrete presentation of the AN. Oral grasping and mouthing of the $\mathrm{AN}$ were preceded by higher levels of activity relative to $\mathrm{AN}$ presentations that resulted in no response. Oral grasping of the AN was preceded by greater variability in activity relative to presentations that resulted in mouthing; in turn, this latter variability was greater than the variability preceding presentations that resulted in no response. However, the level and variabil-

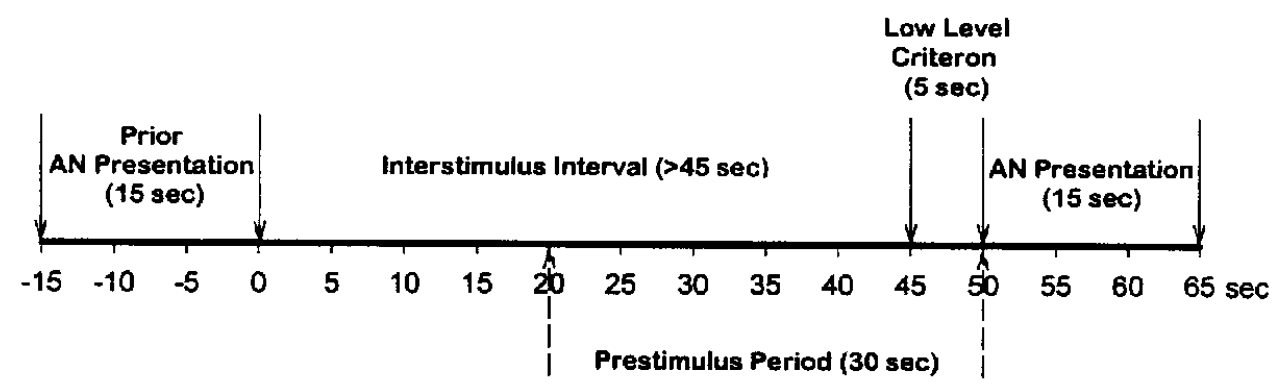

Figure 4. Time line of discrete presentation of the artificial nipple (AN). Solid arrows demarcate experimental time periods, and broken arrows indicate periods used for analysis of overall motor activity. After each AN presentation, an interstimulus interval of at least $45 \mathrm{~s}$ was required before the 5-s low-level criterion could be met, signaling the next AN exposure. A 30-s prestimulus period was extracted from the activity time series for analysis. Time 0 corresponds to the end of the previous AN presentation and the beginning of the current interstimulus interval. 
ity in activity did not differ according to the preceding behavioral response. Collectively, the results from Experiment 2 suggest that mouthing and grasping responses evoked by an AN are facilitated by an increased level of overall activity during the preceding $30 \mathrm{~s}$ and that the progression from mouthing to oral grasping is further facilitated by increased short-term variability in prestimulus activity.

\section{General Discussion}

Continuous presentation of an AN results in higher amplitude motor activity with faster fluctuations. The incidence of mouthing and grasping evoked by the AN cannot directly account for the changes apparent in overall activity of fetuses continuously exposed to the AN. When fetuses are exposed to discrete presentations of the AN, the nature of the response depends on the characteristics of overall activity during the period preceding stimulus presentation. Higher levels of overall activity preceding AN presentation appear to facilitate the expression of both mouthing and oral grasping behavior. Mouthing and, to a greater degree, oral grasping appear to be facilitated by variability in preceding overall activity.

It has been suggested that behavioral organization in the fetus emerges from interactions between simple, independent motor elements resulting in discernible movement patterns (Robinson \& Smotherman, 1988). Although behavioral organization is present in spontaneous fetal motor activity, there are numerous examples of experimental presentations of sensory stimuli to the fetus that enhance fetal behavioral organization and result in the expression of highly coordinated action patterns (e.g., Browne, Robinson, \& Smotherman, 1994; Robinson et al., 1992; Robinson \& Smotherman, 1992a, 1994; Smotherman, Arnold, \& Robinson, 1993; Smotherman \& Robinson, 1987a). By promoting changes in overall motor activity, the presentation of an appropriate stimulus may lead to more interactions between independent motor elements and thereby facilitate the expression of specific action patterns (Robinson \& Smotherman, 1992a).

In the present experiments, the increase in behavioral activation, characterized by an increase in both the level and variability of overall activity, may have potentiated mouthing in response to stimulation of the perioral region by the AN. Mouthing, in turn, permits grasping to occur, so the increase in grasping after periods of increased behavioral activation is not unexpected. However, mouthing and oral grasping were distinguished by the variability in overall activity during the $30 \mathrm{~s}$ preceding presentation of the stimulus but not by the level of overall activity. Increased variability, as measured in this experiment, reflects the temporal clustering of behavioral activation on a short time scale. These conditions, more than general activation, may have facilitated the coordination of mouthing, head movements, and other components of grasping (Robinson et al., 1992) in response to the perioral stimulation provided by the $\mathrm{AN}$.
Results from both Experiment 1 and Experiment 2 implicate variability as an important property of motor activity in characterizing the changes brought about by AN stimulation and in characterizing the periods of activity preceding presentations that resulted in grasping. Although variability has traditionally been considered as a measure of noise or error with little or no functional significance, the notion that variability may be an important component of behavioral expression is gaining support in a dynamic approach to understanding behavior and development (e.g., Thelen \& Smith, 1994). Variability may be both a manifestation of instability near a transition and the process by which behavioral reorganization is accomplished. Increased variability in overall motor activity may reflect a condition of behavioral instability during which an appropriate stimulus (such as a nipple for the rat) can trigger behavioral reorganization and the expression of specific motor patterns (such as mouthing). Furthermore, the clustering of behavioral activation on short time scales, reflected in high-amplitude, short-term fluctuations, may facilitate the coordination of the components of more complex motor patterns (such as oral grasping). Thus, variability in the activation of a developing motor system on a scale of seconds may allow for the emergence of complex movement patterns during behavioral ontogeny.

\section{References}

Becker, L. A., \& Smotherman, W. P. (1996). Manipulation of the dopamine system affects fetal and neonatal responses to an artificial nipple. Developmental Psychobiology, 29, 139-156.

Bekoff, A. (1981a). Embryonic development of chick motor behavior. Trends in Neuroscience, 4, 181-183.

Bekoff, A. (1981b). Embryonic development of the neural circuitry underlying motor coordination. In W. M. Cowen (Ed.), Studies in developmental neurobiology: Essays in honor of Viktor Hamburger (pp. 134-170). New York: Oxford University Press.

Bekoff, A., \& Lau, B. (1980). Interlimb coordination in the 20-day-old rat fetus. Journal of Experimental Zoology, 214, 173-175.

Browne, J. B., Robinson, S. R., \& Smotherman, W. P. (1994). Fetal experience with milk or an artificial nipple alters appetitive and aversive responses to perioral cutaneous stimuli. Behavioral Neuroscience, 108, 606-613.

de Vries, J. I. P., Visser, G. H. A., \& Prechtl, H. F. R. (1982). The emergence of fetal behaviour: I. Qualitative aspects. Early Human Development, 7, 301-322.

de Vries, J. I. P., Visser, G. H. A., \& Prechtl, H. F. R. (1985). The emergence of fetal behaviour: II. Quantitative aspects. Early Human Development, 12, 99-120.

Hamburger, V. (1963). Some aspects of the embryology of behavior. Quarterly Review of Biology, 38, 324-365.

Hamburger, V. (1973). Anatomical and physiological basis of embryonic motility in birds and mammals. In G. Gottlieb (Ed.), Behavioral embryology (pp. 51-76). New York: Academic Press.

Hooker, D. (1952). The prenatal origin of behavior (18th Porter lecture series). Lawrence: University of Kansas Press.

Jenkins, G. M., \& Watts, D. G. (1968). Spectral analysis and its applications. San Francisco: Holden-Day.

Narayanan, C. H., Fox, M. W., \& Hamburger, V. (1971). Prenatal development of spontaneous and evoked activity in the rat. Behavior, 40, 100-134. 
National Institutes of Health. (1986). Guide for the care and the use of laboratory animals (DHEW Publication No. 86-23). Washington, DC: U.S. Government Printing Office.

Oppenheim, R. W. (1981). Ontogenetic adaptations and retrogressive processes in the development of the nervous system and behavior: A neuroembryological perspective. In K. J. Connolly \& H. R. F. Prechtl (Eds.), Maturation and development (pp. 73-109). Philadelphia: Lippincott.

Oppenheim, R. W. (1982). The neuroembryological study of behavior: Progress, problems, perspectives. In R. K. Hunt (Ed.), Current topics in developmental biology: Vol. 17. Neural development, Part III, neuronal specificity, plasticity, and patterns (pp. 257-309). New York: Academic Press.

Oppenheim, R. W. (1984). Ontogenetic adaptations in neural and behavioral development: Toward a more 'ecological' developmental psychobiology. In H. F. R. Prechtl (Ed.), Continuity of neural functions from prenatal to postnatal life (pp. 16-30). Philadelphia: Lippincott.

Preyer, W. (1937). Embryonic motility and sensitivity. Monographs of the Society for Research in Child Development, 2(6, Serial No. 13).

Robertson, S. S. (1985). Cyclic motor activity in the human fetus after midgestation. Developmental Psychobiology, 18, 411-419.

Robertson, S. S., \& Bacher, L. F. (1995). Oscillation and chaos in fetal motor activity. In J. P. Lecanuet, W. P. Fifer, N. A. Krasnegor, \& W. P. Smotherman (Eds.), Fetal development: A psychobiological perspective (pp. 169-189). Hillsdale, NJ: Erlbaum.

Robertson, S. S., Johnson, S. L., Bacher, L. F., Wood, J. R., Wong, C. H., Robinson, S. R., Smotherman, W. P., \& Nathanielsz, P. W. (1996). Contractile activity of the uterus prior to labor alters the temporal organization of spontaneous motor activity in the fetal sheep. Developmental Psychobiology, 29, 667-683.

Robinson, S. R., Hoeltzel, T. C. M., Cooke, K. M., Umphress, S. M., Murrish, D. E., \& Smotherman, W. P. (1992). Oral capture and grasping of an artificial nipple by rat fetuses. Developmental Psychobiology, 25, 543-555.

Robinson, S. R., \& Smotherman, W. P. (1987). Environmental determinants of behavior in the rat fetus: II. The emergence of synchronous movement. Animal Behavior, 35, 1652-1662.

Robinson, S. R., \& Smotherman, W. P. (1988). Chance and chunks in the ontogeny of fetal behavior. In W. P. Smotherman \& S. R. Robinson (Eds.), Behavior of the fetus (pp. 95-115). Caldwell, NJ: Telford Press.

Robinson, S. R., \& Smotherman, W. P. (1992a). The emergence of behavioral regulation during fetal development. In G. Turkewitz (Ed.), Annals of the New York Academy of Sciences: Vol. 662.
Developmental psychobiology (pp. 151-163). New York: New York Academy of Sciences.

Robinson, S. R., \& Smotherman, W. P. (1992b). Fundamental motor patterns of the mammalian fetus. Journal of Neurobiology, $23,1574-1600$.

Robinson, S. R., \& Smotherman, W. P. (1994). Behavioral effects of milk in the rat fetus. Behavioral Neuroscience, 108, 11391149.

Smotherman, W. P., Arnold, H. M., \& Robinson, S. R. (1993). Responses to ecologically relevant stimuli in the rat fetus: Interactive effects of milk and an artificial nipple. Developmen tal Psychobiology, 26, 359-374.

Smotherman, W. P., Richards, L. S., \& Robinson, S. R. (1984). Techniques for observing fetal behavior in utero: A comparison of chemomyelotomy and spinal transection. Developmental Psychobiology, 17, 661-664.

Smotherman, W. P., \& Robinson, S. R. (1987a). Prenatal expression of species-typical action patterns in the rat fetus (Rattus norvegicus). Journal of Comparative Psychology, 101, 190-196.

Smotherman, W. P., \& Robinson, S. R. (1987b). Psychobiology of fetal experience in the rat. In N. A. Krasnegor, E. M. Blass, M. A. Hofer, \& W. P. Smotherman (Eds.), Perinatal development: A psychobiological perspective (pp. 39-60). Orlando, FL: Academic Press.

Smotherman, W. P., \& Robinson, S. R. (Eds.). (1988). Behavior of the fetus. Caldwell, NJ: Telford Press.

Smotherman, W. P., \& Robinson, S. R. (1990). The prenatal origins of behavioral organization. Psychological Science, 1, 97-106.

Smotherman, W. P., \& Robinson, S. R. (1991). Accessibility of the rat fetus for psychobiological investigation. In H. Shair, G. A. Barr, \& M. A. Hofer (Eds.), Developmental psychobiology: New methods and changing concepts (pp. 148-164). London: Oxford University Press.

Smotherman, W. P., \& Robinson, S. R. (1996). The development of behavior before birth. Developmental Psychology, 32, 425-434.

Smotherman, W. P., Robinson, S. R., \& Robertson, S. S. (1988). Cyclic motor activity in the fetal rat (Rattus norvegicus). Journal of Comparative Psychology, 102, 78-82.

Thelen, E., \& Smith, L. B. (1994). A dynamic systems approach to the development of cognition and action. Cambridge, MA: MIT Press.
Received June 27, 1996

Revision received December 4, 1996 Accepted January 7, 1997 